\title{
Phase-sensitive Fourier space imaging of optical Bloch modes
}

\author{
N. Le Thomas and R. Houdré \\ Institut de Photonique et d'Electronique Quantique, Ecole Polytechnique Fédérale de Lausanne (EPFL), Station 3, CH-1015 Lausanne, \\ Switzerland \\ M. V. Kotlyar and T. F. Krauss \\ SUPA, School of Physics and Astronomy, University of St. Andrews, St. Andrews, Fife KY16 9SS, United Kingdom
}

(Received 23 May 2008; published 26 June 2008)

\begin{abstract}
We present a phase-shifting holography method in $k$ space that allows us to investigate the phase and amplitude of optical Bloch waves. The method is based on a Fourier space imaging technique combined with a Mach-Zehnder interferometer. Using a W3 photonic crystal waveguide as a model system, we determine, with high accuracy, both the intensity and the phase of the envelope function of the Bloch modes propagating in such waveguides. We show that local variation of the dielectric map as low as $20 \mathrm{~nm}$ can be clearly observed from the part of the angular spectrum of field located inside the light cone.
\end{abstract}

DOI: 10.1103/PhysRevB.77.245323

PACS number(s): 42.25.Bs, 42.30.Kq, 42.70.Qs, 78.67.-n

\section{INTRODUCTION}

The angular spectrum of wave fields radiated from an object provides a convenient representation of the properties of the object itself. ${ }^{1}$ Such a representation is well suited to the investigation of the optical modes propagating in nanostructures. The experimental determination of this angular spectrum generally consists in recording the scattered intensity as a function of the scattering angle, as, e.g., in x-ray diffraction, electron diffraction, and radioastronomy. For a complete and unequivocal reconstruction of the radiating electromagnetic sources, the knowledge of the intensity and the phase of the fields are both mandatory. Although iterative phase-retrieval algorithms ${ }^{2}$ demonstrated the reconstruction of complex waves from the far-field intensity pattern, i.e., the Fraunhofer pattern, the most popular methods of retrieving the phase information are based on the interference of the scattered modes with a coherent reference wave ${ }^{3}$ and on the link between the intensity propagation and phase gradient of the field. ${ }^{4,5}$ The preference for direct experimental phaseretrieval techniques is due to the convergence and noise sensitivity issues inherent in iterative algorithms. ${ }^{4}$

The angular spectrum representation, which is, for instance, written

$$
U(x, y, z)=\int_{-\infty}^{+\infty} \int_{-\infty}^{+\infty} a(u, v) e^{i k_{0}\left(u x+v y-\sqrt{k_{0}^{2}-u^{2}-v^{2}} z\right)} d u d v
$$

for a scalar wave field $U$ propagating in a half-space, with $k_{0}$ the free-space wave vector, gives direct access to the transverse spatial frequencies $u$ and $v$ of the radiating current or vector potential induced in the object. ${ }^{6}$ The spectral amplitudes $a(u, v)$ of each plane-wave mode in the angular spectrum representation can be experimentally retrieved either in the near field or in the far field. Plane-wave modes, which have different spectral amplitudes, are per nature spatially separated in the far field. It follows that each spatial harmonics of a radiated wave field can be investigated separately and their directions of propagation uniquely determine the transverse spatial frequencies that belong to the radiating field. As a result, such a representation provides a simple and fundamental analysis of the physical nature of the fields excited inside the object without carrying out detailed calculations. Imaging the intensity and the phase of the angular spectrum of modes excited in photonic nanostructures is therefore an important task pioneered by Kuipers and co-workers ${ }^{7,8}$ in the near field with phase-sensitive scanning near-field optical microscope (SNOM). Such a phasesensitive technique that can recover the complex field by probing the near field with a tip gives, in principle, access to the $k$ space with unprecedented $k$ bandwidth. As an alternative, we have recently developed a far-field technique referred to as Fourier space imaging. ${ }^{9}$ Fourier space imaging monitors the mode pattern in the back focal plane of a microscope objective, i.e., it directly images the Fraunhofer pattern. ${ }^{10,11}$ This technique, in principle, is limited to modes operating above the light line, although we have shown that it can be extended considerably below the light line using "super-resolution" techniques. ${ }^{9,12}$

Unlike in scanning near-field techniques, the mode field is not disturbed by the presence of a scanning tip nor does it require any sophisticated numerical reconstruction of the vectorial transfer function of the tip or numerical postprocessing to extract the desired information. Instead, Fourier space imaging is accurate, fast, and direct. The missing element, however, is the availability of phase information, which is introduced here; by adding a Mach-Zehnder interferometer into the Fourier space imaging setup, we demonstrate that both the $k$ vector and the phase of optical modes can be monitored at the video speed of the recording charge coupled device (CCD) camera. As a result, the complex amplitude of the angular spectrum of components of Bloch modes propagating in photonic crystal waveguides can be determined.

Photonic crystal structures are ideal model objects because their electromagnetic properties in the near field can, in principle, be completely predicted theoretically, with, e.g., three-dimensional finite difference time domain computation. From this point of view, the photonic crystals provide perfect reference objects, with well-defined Bloch modes, whose angular $(\omega)$ and spatial $(k)$ frequency spectra cover a large bandwidth. As a proof of principle, we use here a multimode 


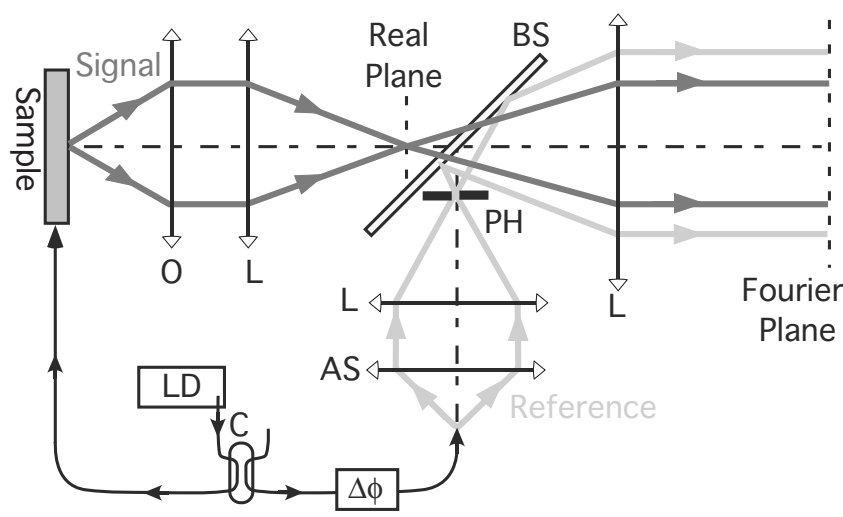

FIG. 1. Experimental setup. LD-tunable laser diode, $\mathrm{C}-2$ $\times 2$ coupler, $\Delta \phi$ - phase delay, $\mathrm{O}$-collecting microscope objective $(\mathrm{NA}=0.9), \mathrm{AS}$ - aspheric lens, L-lenses, BS—beam splitter, and $\mathrm{PH}-$ pinhole.

PhC waveguide, whose different modes exhibit angular spectrum with well-defined phases of 0 or $\pi$.

\section{INTENSITY PATTERN IN THE FOURIER SPACE}

The setup is schematically shown in Fig. 1. Light from an external cavity diode laser that is tunable between 1.48 and $1.66 \mu \mathrm{m}$ is coupled into a $2 \times 2$ single mode fiber coupler in order to create a Mach-Zehnder interferometer. In the signal arm the Bloch modes of the photonic crystal structure are selectively excited by the narrow bandwidth $(\Delta \lambda=1 \mathrm{pm})$ laser source via standard lens fibers and integrated access ridge waveguides. A home built infrared microscope described in Ref. 9 generates the Fourier transform of the object in the back focal plane of a high numerical aperture $(\mathrm{NA}=0.9) \mathrm{mi}-$ croscope objective. This technique provides an experimental two-dimensional (2D) intensity plot, in $k$ space, of the field emitted from the surface of the sample. As a result the phase velocities and the dispersion curve of the Bloch modes are directly and uniquely inferred, as shown in Fig. 2(b). The $k$-space signal corresponds to the Bloch mode spatial frequencies located inside the bandwidth of the imaging setup. In the reference arm, the light provided by the second output port of the coupler is focused onto a pinhole and is collimated to provide an aberration-free plane wave over the entire back focal plane of the collecting microscope objective. The fields from the reference arm and the signal arm are combined and the resulting interferogram is measured on an infrared CCD detector located in the Fourier plane.

The object, a W3 waveguide [Fig. 2(a)], ${ }^{13}$ is defined by three lines of missing holes in a 2D triangular lattice of holes etched in a dielectric planar waveguide. The triangular lattice parameter, hole diameter, and total length are $a=0.40 \mu \mathrm{m}$, $d=0.27 \mu \mathrm{m}$, and 128 and $24 \mu \mathrm{m}$ for Figs. 2 and 3, respectively. The GaInAsP/InP planar waveguide that provides optical confinement in the $z$ direction has an effective index of $n_{\text {eff }}=3.25$ and is single mode in the frequency range of interest.

Figure 2 reveals the nature and the difference of the information provided by the real and Fourier space imagings, respectively. As shown in Fig. 2(c), the near infrared realspace image of the field radiated from the surface of the sample exhibits two parallel lines of periodic nodes mainly located at the first line of holes that borders the W3 waveguide. The periodicity of the intensity pattern reveals the presence of nonzero spatial frequencies oriented along the $x$ axis. When the excitation wavelength increases, the spacing between the periodic nodes of the fields decreases in agreement with the variation of the dispersion curve [Fig. 2(b)]. The observed spatial modulation comes from an interference effect between forward and backward propagating Bloch
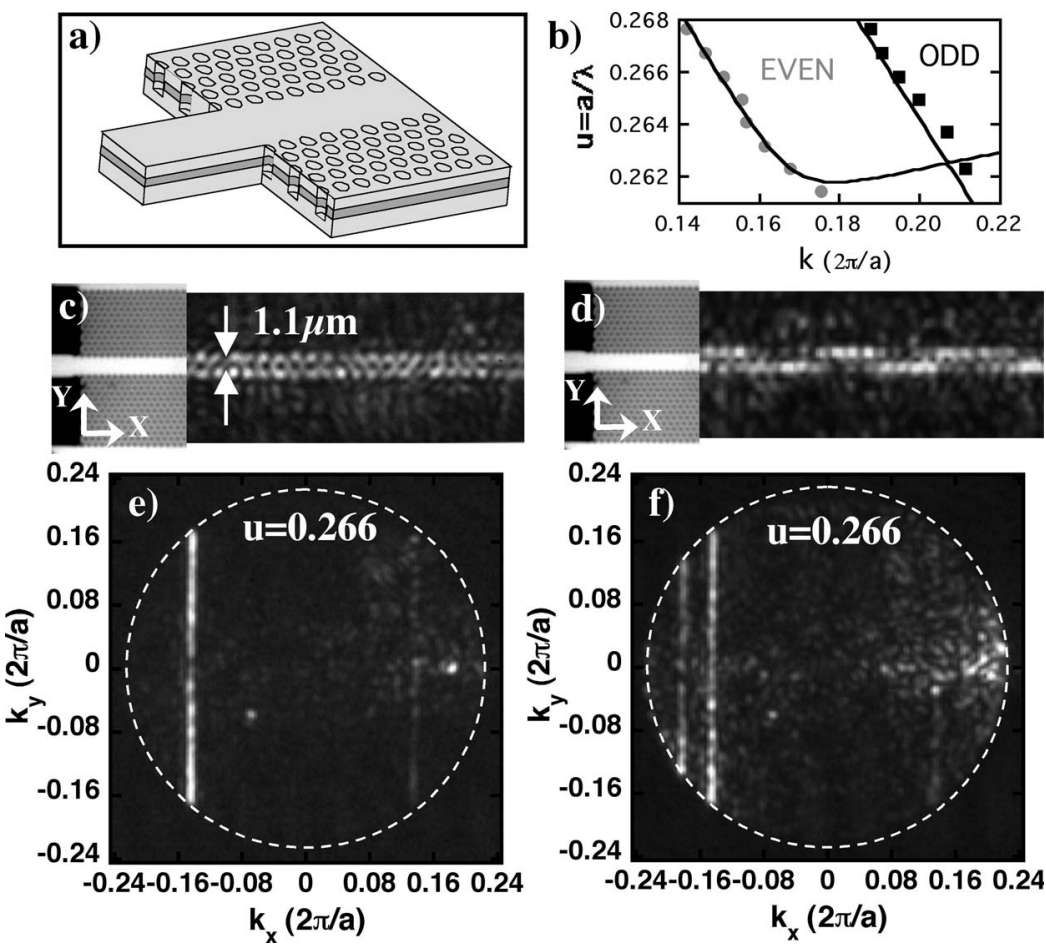

FIG. 2. (a) Schematic of the planar W3 PhC waveguide. (b) Theoretical (line) and experimental (dots) dispersion curves. [(c) and (b)] Real space of the infrared field propagating in a W3 $\mathrm{PhC}$ waveguide without and with mode beating, respectively, [(e) and (f)] corresponding Fourier space images. 


\section{INTERFEROGRAM}

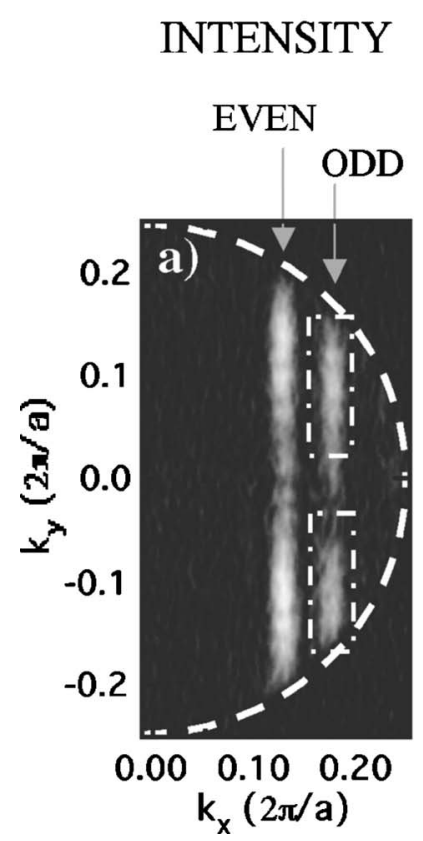

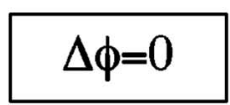

EVEN

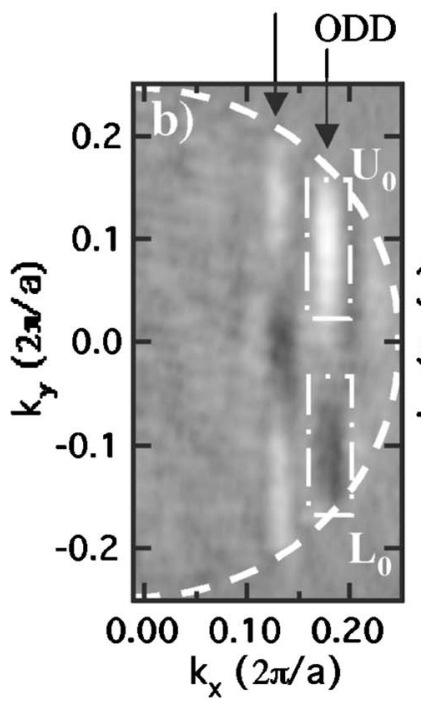

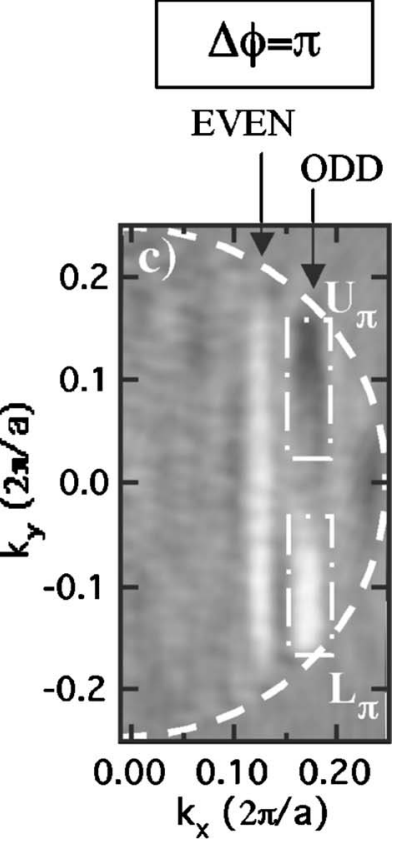

FIG. 3. (a) Standard Fourier space image of the W3 even and odd modes (the reference arm off). [(b) and (c)] Corresponding Fourier space interferogram for 0 (right) and $\pi$ (left) relative phase $\Delta \phi$ between the signal and the reference (the reference arm on). modes. The backward propagation is induced by reflection at the output cleaved facet of the sample. Such kind of standing-wave pattern has already been used to determine the modulus of the wave vector of Bloch modes. ${ }^{14}$ Note that when only one Bloch mode propagates in the structure and only one of its spatial harmonics is detected by the setup, a homogeneous unstructured pattern is observed in real space. It follows that no information about the wave vector can be directly retrieved in the real-space image if the sample approaches perfection.

In Fig. 2(d), a slight transverse shift of the input lensed fiber compared to the center of the access ridge waveguides induces a drastic change in the interference pattern of the image. This observation is associated with a mode beating, which results from the multimode character of the W3 waveguide. Since the access ridge waveguide supports multiple modes, the lateral translation of the fiber allows the additional excitation of uneven modes. The beating length gives access to the modulus of the spatial frequency separation between the two Bloch modes, i.e., their $\Delta k$, but not to their actual $k$ values.

In Fig. 2(e), in contrast to the real-space image, these $k$ values are unambiguously determined without the need for interference effects. The far-field pattern imaged in $k$ space [Fig. 2(e)] corresponding to Fig. 2(c) is composed of a dominant straight line located at $-k_{x}$ and perpendicular to the propagation direction of the mode, which is similar to signal retrieved with phase-sensitive SNOM technique. ${ }^{8}$ Determining the $k_{x}$ position of the diffracted lines at different wavelengths gives access to the experimental dispersion curves plotted in Fig. 2(b). The experimental data are well fitted for each mode, the even (circles) and the odd (squares), by a 2D plane-wave expansion model with a filling factor $f \sim 0.3$ as input parameter. The weaker line symmetrically located at $+k_{x}$ is attributed to the reflected wave from the cleaved facet

of the output waveguide..$^{15}$ The interferences produced by these two distinct spatial frequencies are responsible for the periodic nodes measured in Fig. 2(c).

In connection with the mode beating observed in Fig. 2(d), an additional line appears at $-k_{x}$ in the $k$ space when the input fiber is slightly laterally shifted [Fig. 2(f)]. As well as the spacing between the forward and backward propagating modes, the spacing between the two lines at $-k_{x}$ in Fig. 2(f) is directly related to the period of the beating (i.e., twice the coupling length). From the comparison between the experimental and theoretical dispersion curves [Fig. 2(b)], we can infer that the two lines correspond to even and odd modes, respectively, as will be confirmed by the interferometric measurement in Fourier space.

Figure 2 demonstrates that the $k$-space imaging is very convenient for assessing the algebraic value of the wave vector and for analyzing the nature of the different modes. It also clearly enables the retrieval of more information than from conventional real-space imaging. However, the complex weight of each components of a Bloch wave, or the phase of the envelope of the field, in particular here along the confinement $y$ direction is still undetermined.

\section{AMPLITUDE OF THE FAR-FIELD IN THE FOURIER SPACE}

Along the $y$ direction, the condition of translation invariance, which comes from the $\mathrm{PhC}$ periodicity, is relaxed. As a result the $k_{y}$ angular distribution evolves from a periodic discrete distribution of Dirac function into a more complex continuous envelope function. Using our interferometric technique, the intensity profile $I_{s}\left(k_{x}, k_{y}\right)=\left|E_{s}\left(k_{x}, k_{y}\right)\right|^{2}$ and the phase $\phi_{s}\left(k_{x}, k_{y}\right)$ of such an envelope are completely determined. In Figs. 3(b) and 3(c), the measured interferograms $S_{0}\left(k_{x}, k_{y}\right)$ and $S_{\pi}\left(k_{x}, k_{y}\right)$ in the Fourier space for a 0 and $\pi$ 

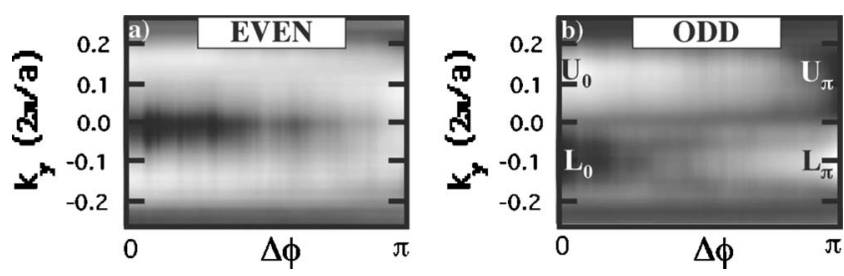

FIG. 4. [(a) and (b)] Variation of the $k$-space profile of the even and odd modes, respectively, versus the relative phase $\Delta \phi$ between the signal and the reference.

phase delay between the radiative Bloch waves and the reference beam, respectively, reveal the distinct nature of the envelope for the even and the odd modes. In contrast to the noninterfering case [Fig. 3(a)] where the background level is around zero intensity (dark color), the background reference plane $R$ of the interferograms is set at the intensity of the reference beam where no signal from the sample is present (gray color). For each mode, the profile of the interferogram along $k_{y}$ oscillates between dark [e.g., the region labeled $L_{0}$ in Fig. 3(b)] and bright [e.g., the region labeled $U_{0}$ in Fig. $3(b)]$ intensities on either side of $R$, which is a clear signature of a spatial variation of the phase. The symmetry of these profiles also corresponds to the theoretically expected modal symmetry: The line profile is symmetric around $k_{y}=0$ for the even mode and antisymmetric for the odd mode. Figures $3(b)$ and 3(c) show that a $\pi$ shift of the relative phase $\Delta \phi$ between the signal and the reference over all the $k$ space induces an antisymmetrical switch of the maximum $\left(U_{0}\right.$ and $\left.L_{\pi}\right)$ and minimum $\left(L_{0}\right.$ and $\left.U_{\pi}\right)$ of the profile along $k_{y}$ for the odd mode. Such a switch between, e.g., $U_{0}$ and $U_{\pi}$ is characteristic of a constant phase of the field envelope in the $U$ regions. In Fig. 4, the plot of the angular profile along $k_{y}$ as a function of a continuous variation of $\Delta \phi$ from 0 to $\pi$ confirms that the two lobes of the odd mode observed in Fig. $3(a)$ are in antiphase.

The different interferograms provide a quantitative determination of the envelope function of the angular spectrum of each mode. Without the reference beam, the signal is proportional to $I_{s}\left(k_{x}, k_{y}\right)$. With the reference beam of constant intensity $I_{r}\left(k_{x}, k_{y}\right)=I_{r}=\left|E_{r}\right|^{2}$ and spatially constant phase $\phi_{r}\left(k_{x}, k_{y}\right)=\phi_{r}$, the interference process in the Fourier space induces an interferogram with the intensity pattern $S_{\phi_{r}}\left(k_{x}, k_{y}\right)$ given by

$$
S_{\phi_{r}}(k)=\left|E_{s}(k)\right|^{2}+\left|E_{r}\right|^{2}+2 E_{r}^{*} E_{s}(k) \cos \left[\phi_{s}(k)-\phi_{r}\right],
$$

where $k=\left(k_{x}, k_{y}\right)$ and $E_{r}^{*}$ is the complex conjugate of $E_{r}$. The control of the relative phase $\Delta \phi=\phi_{s}\left(k_{x}, k_{y}\right)-\phi_{r}$ gives a direct access to the phase of the different spectral components of the radiated signal. The phase of the field can be determined from three independent interferograms, for instance, $\phi_{r}=0$, $\phi_{r}=\pi$, and $\phi_{r}=\pi / 2$. From Eq. (2) we obtain the two following equations that uniquely define the phase $\phi_{s}(k)$ modulo $2 \pi$ :

$$
\phi_{s}(k)=\arctan \left(\frac{S_{0}(k)-S_{\pi / 2}(k)}{S_{\pi / 2}(k)-S_{\pi}(k)}\right)-\frac{\pi}{4},
$$

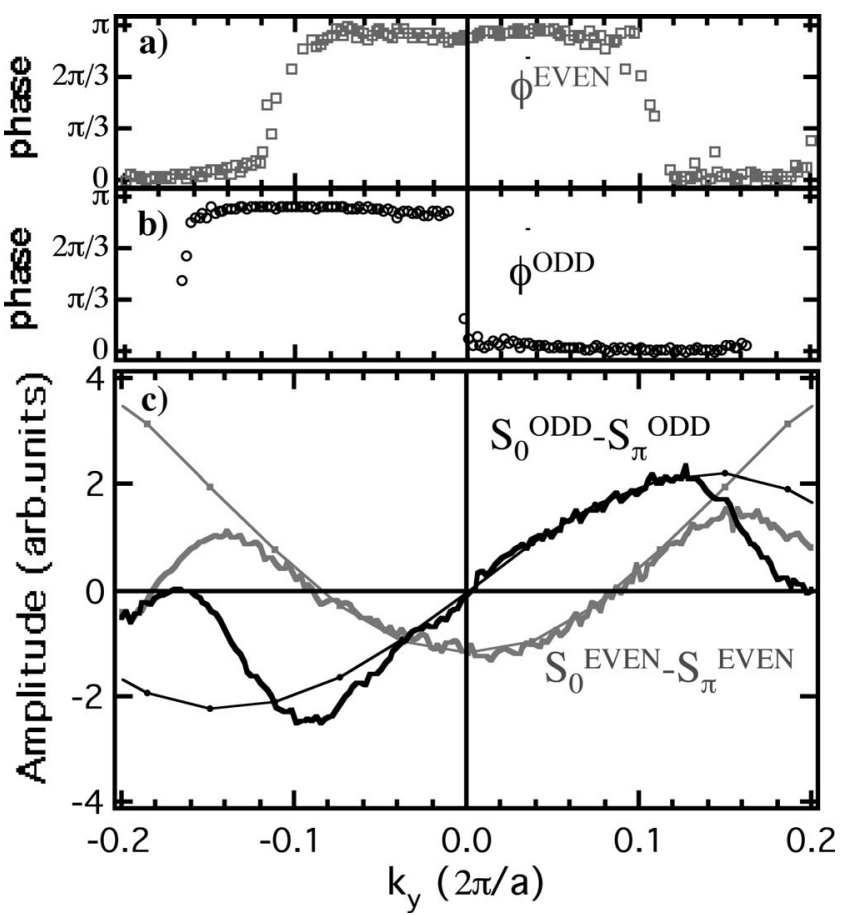

FIG. 5. [(a) and (b)] Phase of the even and odd modes, respectively. (c) Experimental (thick lines) and theoretical (thin lines with dots) amplitudes of the envelope of the even (dark) and odd (white) modes.

$$
S_{0}(k)-S_{\pi}(k)=4 E_{r}^{*} E_{s}(k) \cos \left[\phi_{s}(k)\right] .
$$

As a result, Figs. 5(a) and 5(b) show that the experimental phases of the even and odd modes switch both from 0 to $\pi$, as expected for a one-dimensional waveguide. The phase discontinuities, observed in $k$-space points where the field amplitude vanishes, correspond to steplike topological singularities of the wave fronts of the fields. ${ }^{16}$ The wave field has an undefined phase at $k$ points where such phase singularities take place. In Fig. 5(c), the experimental amplitudes of the angular spectrum $E_{s}(k) \cos \left[\phi_{s}(k)\right]$ of the Bloch mode are directly deduced from Eq. (3) and interferograms such as in Figs. 3(b) and 3(c). The theoretical amplitudes, obtained from 2D plane-wave calculation using a supercell technique, reproduce the experimental data remarkably well, which confirms that the leaky nature of the modes propagating in planar waveguides with core thickness of $500 \mathrm{~nm}$ does not affect the angular spectrum of the ideal 2D Bloch mode ${ }^{17}$ and that equivalent current methods are well adapted to describing the radiative properties of planar structures. ${ }^{18}$ The spatial filtering in an intermediate real-space imaging plane used to remove background noise speckles is responsible for the smooth cutoff at the pupil of the collecting microscope objective. Note that all the residual optical aberrations of the setup may be digitally corrected if needed. ${ }^{19}$

\section{ANALYTIC CONTINUATION IN THE FOURIER SPACE}

The knowledge of the $k$ spectrum over the pupil of the collecting lens is, in principle, sufficient to mathematically 


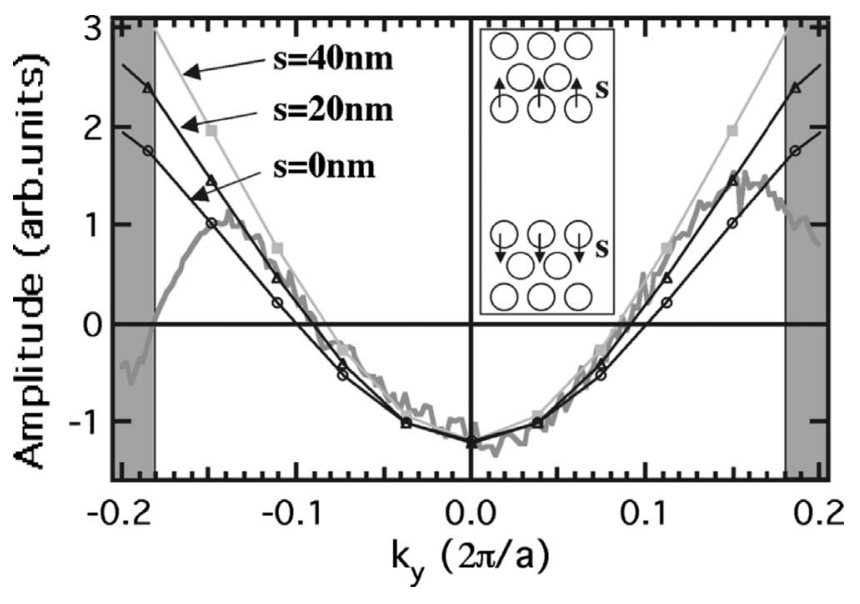

FIG. 6. Comparison between the experimental (thick lines) and theoretical (thin lines with dots) amplitudes of the envelope of the even mode for different hole shift parameters $s$. The gray regions correspond to the light cone. The inset highlights the shifted holes around the W3 line defect.

determine the entire spectrum via analytic continuation. ${ }^{20,21}$ However, in practice the analytical continuation procedure, for instance, with the use of prolate functions ${ }^{22}$ or maximum entropy methods, ${ }^{23}$ is an improperly posed problem due to the presence of noise. ${ }^{24,25} \mathrm{As}$ a result, the enhancement of the spatial frequency bandwidth is drastically limited with enhancement factors not better than $\sim 1.5$. Instead of using the ill-conditioned mathematical analytic continuation procedure, it is nevertheless possible to take advantage of the analytic nature of the problem: As the part of the $k$ space signal of the field measured in the pupil of the collecting lens determines uniquely the entire $k$-space spectrum of the field; any variation of the out-bandwidth spectrum will inevitably impact the $k$-space signal recorded inside the bandwidth of the setup. Of course, the repercussion of any modification of the out-bandwidth $k$-space signal can be observed only if it overcomes the in-bandwidth incertitude related to the noise.

To quantify such an impact we have calculated the theoretical far field of W3 waveguides subject to a slight variation of their dielectric map. As an example the first line of holes on each side of the core of the waveguide has been transversally shifted by an amount $s$ of either 20 or $40 \mathrm{~nm}$ (see inset of Fig. 6). In Fig. 6, the corresponding calculated far-field spectra are compared with the experimental signal. It can be seen that the departure from the experimental data $(s=0)$ can already be observed for $s=20 \mathrm{~nm}$. We conclude that the current experimental noise level allows us to be sensitive to spatial shifts of the holes as small as $\sim 20 \mathrm{~nm}$. Such a modification of the far field from positional variation of the holes that border the $\mathrm{PhC}$ defect was previously applied to explain and optimize the quality factor of L3 cavities by Akahane et al. $^{26}$ (Note that the transverse pattern of the W3 waveguide mimics the L3 cavity if the fundamental mode is considered.) Here we show quantitatively, directly, and without cumbersome data inversion procedures that the signal measured in $k$ space follows nanometric modification of the dielectric map. Our approach is particularly suited to optimize the emission diagram of $\mathrm{PhC}$ based light sources or to investigate and identify with high accuracy the frequency spectrum of the out-of-plane losses. In addition the main source of noise comes here from speckles induced by disorder in the sample. Such a noise is partly attenuated by the filtering procedure in an intermediate imaging plane however to the detriment to the spatial frequency bandwidth as mentioned above. Such a noise can nevertheless, in principle, be suppressed and then the shot noise will be the ultimate contribution that limits the accuracy.

\section{CONCLUSION}

In conclusion, we have imaged the complex amplitude of the angular spectrum of Bloch modes propagating in photonic crystal waveguides and thereby demonstrated the capability of our phase-sensitive Fourier imaging technique to characterize nanophotonic structures. This demonstration paves the way for the investigation of exotic light beams radiated from other nanostructures, such as beams that possess a quantized orbital angular momentum. ${ }^{27}$ In principle, the rich variety of designs offered by $2 \mathrm{D}$ photonic crystal engineering allows the generation of such helically phased beams, which are appealing, e.g., for quantum information processing, microparticle manipulation, and cold atom trapping. With the phase-sensitive Fourier imaging technique, their signature should consist in spiral intensity patterns. ${ }^{27}$

\section{ACKNOWLEDGMENTS}

This project was supported by the European network of excellence Epixnet under Grant No. IST-004525, Funfox under Grant No. IST-004582, the Swiss Quantum Photonics National Center for Competence in Research, and the COST action P11.
${ }^{1}$ M. Born and E. Wolf, Principles of Optics (Cambridge University Press, Cambridge, 1999).

${ }^{2}$ J. R. Fienup, J. Opt. Soc. Am. A 4, 118 (1987); J. R. Fienup, Opt. Lett. 3, 27 (1978).

${ }^{3}$ D. Gabor, Nature (London) 161, 777 (1948).

${ }^{4}$ N. Nakajima, Phys. Rev. Lett. 98, 223901 (2007).

${ }^{5}$ K. A. Nugent, D. Paganin, and T. E. Gureyev, Phys. Today 54, 27 (2001).
${ }^{6}$ L. Mandel and E. Wolf, Optical Coherence and Quantum Optics (Cambridge University Press, Cambridge, 1995).

${ }^{7}$ M. L. M. Balistreri, H. Gersen, J. P. Korterik, L. Kuipers, and N. F. van Hulst, Science 294, 1080 (2001).

${ }^{8}$ R. J. P. Engelen, Y. Sugimoto, H. Gersen, N. Ikeda, K. Asakawa, and L. Kuipers, Nat. Phys. 3, 401 (2007).

${ }^{9}$ N. Le Thomas, R. Houdré, M. V. Kotlyar, D. O’Brien, and T. F. Krauss, J. Opt. Soc. Am. B 24, 2964 (2007). 
${ }^{10}$ J. W. Goodman, Introduction to Fourier Optics, 3rd ed. (Roberts \& Company, Englewood, Colorado, 2005).

${ }^{11}$ B. Hecht, H. Bielefeldt, L. Novotny, Y. Inouye, and D. W. Pohl, Phys. Rev. Lett. 77, 1889 (1996).

${ }^{12}$ N. Le Thomas, R. Houdré, L. H. Frandsen, J. Fage-Pedersen, A. V. Lavrinenko, and P. I. Borel, Phys. Rev. B 76, 035103 (2007).

${ }^{13}$ S. Olivier, M. Rattier, H. Benisty, C. Weisbuch, C. J. M. Smith, R. M. De La Rue, T. F. Krauss, U. Oesterle, and R. Houdré, Phys. Rev. B 63, 113311 (2001).

${ }^{14}$ M. Loncar, D. Nedeljkovic, T. P. Pearsall, J. Vučković, A. Scherer, S. Kuchinsky, and D. C. Allan, Appl. Phys. Lett. 80, 1689 (2002).

${ }^{15}$ This line almost disappears when an antireflection coating is deposited on the cleaved facets of the sample.

${ }^{16}$ G. Popescu and A. Dogariu, Phys. Rev. Lett. 88, 183902 (2002).

${ }^{17}$ L. C. Andreani, Phys. Status Solidi B 234, 139 (2002).
${ }^{18}$ S. A. Schelkunoff, Phys. Rev. 56, 308 (1939).

${ }^{19}$ T. Colomb, E. Cuche, F. Charrière, J. Kühn, N. Aspert, F. Montfort, P. Marquet, and C. Depeursinge, Appl. Opt. 45, 851 (2006).

${ }^{20}$ B. R. Frieden, Prog. Opt. 9, 311 (1971).

${ }^{21}$ A. Papoulis, IEEE Trans. Circuits Syst. 22, 735 (1975).

${ }^{22}$ V. N. Beskrovnyy and M. I. Kolobov, Phys. Rev. A 71, 043802 (2005).

${ }^{23}$ S. F. Gull and G. J. Daniell, Nature (London) 272, 686 (1978).

${ }^{24}$ M. Bertero, C. De Mol, and G. A. Viano, J. Math. Phys. 20, 509 (1979).

${ }^{25}$ D. L. Donoho, I. M. Johnstone, J. C. Hoch, and A. S. Stern, J. R. Stat. Soc. Ser. B (Methodol.) 54, 41 (1992).

${ }^{26}$ Y. Akahane, T. Asano, B.-S. Song, and S. Noda, Nature (London) 425, 944 (2003).

${ }^{27}$ M. Padgett, J. Courtial, and L. Allen, Phys. Today 57, 35 (2004). 